\title{
Characterization of the time-frequency parameters inherent in the radiation of semiconductor heterolasers using interferometric technique
}

\author{
Alexandre S. Shcherbakov ${ }^{1)}$, Pedro Moreno Zarate ${ }^{1)}$, Joaquin Campos Acosta ${ }^{2)}$,
} Yurij V. Il'n ${ }^{3)}$, Il'ya S. Tarasov ${ }^{3)}$.

1) National Institute for Astrophysics, Optics and Electronics, Puebla, 72000, Mexico.

Phones: 52 (222) 266 3100, ext. 2205; E-mail: alex@inaoep.mx , pemzamx@gmail.com

2) CSIC-Institute for Applied Physics, Serrano 144, Madrid, 28006, Spain.

Phone: 34 (91) 561 8806, ext. 206; E-mail: joaquin.campos@ifa.cetef.csic.es

3) A.F.Ioffe Physical-Technical Institute of the Russian Academy of Sciences, Polytechnicheskaya St. 26, Saint-Petersburg, 194021, Russian Federation.

Phone: 7 (812) 2927379 or 292 7997; E-mail: ptk@mail.ioffe.ru , tarasov@hpld.ioffe.ru

\begin{abstract}
The specific approach to characterizing the train-average parameters of low-power picosecond optical pulses with the frequency chirp, arranged in high-repetition-frequency trains, in both time and frequency domains is elaborated for the important case when semiconductor heterolasers operate in the active mode-locking regime. This approach involves the joint Wigner time-frequency distributions, which can be created for those pulses due to exploitation of a novel interferometric technique under discussion. Practically, the InGaAsP/InP-heterolasers generating at the wavelength $1320 \mathrm{~nm}$ were used during the experiments carried out and an opportunity of reconstructing the corresponding joint Wigner time-frequency distributions was successfully demonstrated.
\end{abstract}

Key words: Wigner time-frequency distribution, semiconductor heterolaser, pulse parameters, interferometric technique.

\section{INTRODUCTION}

We present an approach to the characterization of low-power bright picosecond optical pulses with an internal frequency modulation in both time and frequency domains in practically important case of exploiting semiconductor heterolasers operating in near-infrared range in the active mode-locking regime ${ }^{\mathbf{1}}$. This approach uses the joint Wigner time-frequency distributions, which can be found for this regime due to involving an original interferometric technique under consideration. In so doing, the modified scanning Michelson interferometer was chosen for shaping the fieldstrength auto-correlation functions peculiar to the pulsed infrared light radiation. We exploit the key features of a new experimental technique for accurate and reliable measurements of the train-average temporal width and the frequency chirp associated with picosecond optical pulses in high-repetition rate pulse trains. This technique is founded on an ingenious algorithm elaborated specially for the advanced optical metrology, which makes possible constructing the joint Wigner distributions for describing the above-listed parameters of optical pulses. The InGaAsP/InP-heterolasers, operating at $1320 \mathrm{~nm}$ range, were exploited during the experiments carried out. When the optical signal consisted of contiguous pulses with the repetition frequency close to $1 \mathrm{GHz}$, due to operating semiconductor heterolasers in the active mode-locking regime, typical pulse train-average auto-correlation function had been characterized by temporal widths in picosecond range. The accuracy of similar measurements increased with growth of the repetition frequency due to rising in a number of samples.

\section{THE JONT TIME-FREQUENCY DISTRIBUTION}

This consideration is directly related to the analysis of signals, which are non-stationary in behavior in time and frequency domains. The main problem here is connected with finding a joint function of both the time and the frequency, i.e. some distribution, which will be able to describe the energy density or the intensity of an arbitrary

Photonics North 2009, edited by Réal Vallée, Proc. of SPIE Vol. 7386, 73862H

(c) 2009 SPIE · CCC code: 0277-786X/09/\$18 · doi: 10.1117/12.838469 
signal in terms of time and frequency simultaneously. Ideally, it would be an analytic function of two arguments having properties of the density function, which characterizes the energy, or intensity, of a signal at unity time interval as well as at unity frequency interval. Together with this, it should be noted that time-frequency analysis exhibits a row of peculiarities; for example, it is governed by the uncertainty principle. Introducing similar joint time-frequency distribution $\mathbf{P}(\mathbf{t}, \omega)$, one can write
a) $\int_{-\infty}^{\infty} \mathbf{P}(\mathbf{t}, \omega) \mathrm{d} \omega=|\mathbf{A}(\mathbf{t})|^{2}$
b) $\int_{-\infty}^{\infty} P(t, \omega) d t=|S(\omega)|^{2}$,

where $\mathbf{A}(\mathbf{t})$ and $\mathbf{S}(\omega)$ are the amplitude of a determined signal and its Fourier transform, respectively, so that

$$
S(\omega)=\frac{1}{2 \pi} \int_{-\infty}^{\infty} A(t) \exp (-i \omega t) d t
$$

The total energy can be determined as

$$
\mathbf{W}_{0}=\int_{-\infty}^{\infty} \int_{-\infty}^{\infty} \mathbf{P}(t, \omega) d \omega d t=\int_{-\infty}^{\infty}|\mathbf{A}(t)|^{2} \mathbf{d} t=\int_{-\infty}^{\infty}|S(\omega)|^{2} \mathrm{~d} \omega
$$

if the conditions for existing both the corresponding one-dimensional distributions are satisfied. It follows from the basic mathematical principles that an uncertainty principle characterizes the fundamental coupling between a rootmean-square deviation of some function and a root-mean-square deviation of Fourier-transfom peculiar to the same function. In particular case of a pair including $\mathbf{A}(\mathbf{t})$ and $\mathbf{S}(\omega)$, these root-mean-square deviations in time and frequency domains can be determined by
a) $\tau_{\mathrm{SA}}=\sqrt{\mathbf{T}_{2}-\left(\mathbf{T}_{1}\right)^{2}}$,
b) $\mathbf{T}_{\mathbf{n}}=\mathbf{W}_{\mathbf{0}}^{-1} \int_{-\infty}^{\infty} \mathbf{t}^{\mathbf{n}}|\mathbf{A}(\mathbf{t})|^{2} \mathbf{d t}$,
a) $\omega_{S A}=\sqrt{\omega_{2}-\left(\omega_{1}\right)^{2}}$,
b) $\omega_{n}=W_{0}^{-1} \int_{-\infty}^{\infty} \omega^{n}|S(\omega)|^{2} d \omega$.

The corresponding uncertainty principle is given by the realation $\tau_{\mathbf{S A}} \cdot \omega_{\mathbf{S A}} \geq \mathbf{1 / 2}$. In context of the determined functions, this relation means that the determined function and its Fourier transform cannot have simultaneously arbitrary small widths. In its turn, in the signal analysis, where $\tau_{\mathbf{S A}}$ has a meaning of the root-mean-square time duration and $\omega_{\mathbf{S A}}$ has a meaning of the root-mean-square spectral width, this fact speaks that the signal and its spectrum cannot have simultaneously arbitrary small widths as well. There are a few options to choose a concrete form of the joint time frequency distribution. In particular, one can take the Wigner time-frequency distribution, i.e. assume that $\mathbf{P}(\mathbf{t}, \omega)=\mathbf{W}(\mathbf{t}, \boldsymbol{\omega})$, which is given by, see for example ${ }^{2}$

$$
W(t, \omega)=\frac{1}{2 \pi} \int_{-\infty}^{\infty} A^{*}\left(t-\frac{\tau}{2}\right) \exp (-i \tau \omega) A\left(t+\frac{\tau}{2}\right) d \tau=\frac{1}{2 \pi} \int_{-\infty}^{\infty} S^{*}\left(\omega-\frac{\theta}{2}\right) \exp (i \mathrm{t} \theta) S\left(\omega+\frac{\theta}{2}\right) d \theta
$$

The kernel of this distribution depends on the product of its arguments. Theoretically, the Wigner distribution has an infinite resolution in time due to absence of averaging over any finite time interval. Moreover, for finite lag length, it has an infinite frequency resolution. Together with this, the Wigner distribution being quadratic in nature is able to introduce various cross terms for a multi-component signal. 


\section{LOCAL AUTO-CORRELATION APPROACH}

Here, the relation between the spectrum density and the auto-correlation function is considered. This approach generalizes such a relation, and it is based on exploiting so-called local auto-correlation function ${ }^{2}$. The key point is connected with the following representation for the spectrum density

$$
|S(\omega)|^{2}=\left|\frac{1}{\sqrt{2 \pi}} \int_{-\infty}^{\infty} \mathbf{A}(\mathbf{t}) \exp (-\mathbf{i} \omega t) d t\right|^{2}=\frac{1}{2 \pi} \int_{-\infty}^{\infty} \int_{-\infty}^{\infty} A^{*}\left(t^{\prime}\right) A(t) \exp \left[i \omega\left(t^{\prime}-t\right)\right] d t^{\prime} d t
$$

Using the substitutions: $\tau=\mathbf{t}-\mathbf{t}^{\prime}$ and $\mathbf{d} \tau=-\mathbf{d} \mathbf{t}^{\prime}$, we obtain $\mathbf{t}^{\prime} \rightarrow \pm \infty$ and $\tau \rightarrow \mp \infty$, so that

$$
|S(\omega)|^{2}=\frac{1}{2 \pi} \int_{-\infty}^{\infty} \int_{-\infty}^{\infty} A^{*}(t-\tau) A(t) \exp [-i \omega \tau] d \tau d t=\frac{1}{2 \pi} \int_{-\infty}^{\infty} R(\tau) \exp [-i \omega \tau] d \tau,
$$

where the auto-correlation function $\mathbf{R}(\tau)$ is defined by

$$
\mathbf{R}(\tau)=\int_{-\infty}^{\infty} \mathbf{A}^{*}(t-\tau) \mathbf{A}(\mathbf{t}) \mathbf{d} \mathbf{t}=\int_{-\infty}^{\infty} \mathbf{A}^{*}(\mathbf{t}) \mathbf{A}(\mathbf{t}+\tau) \mathbf{d} \mathbf{t}=\int_{-\infty}^{\infty} \mathbf{A}^{*}\left(\mathbf{t}-\frac{\tau}{2}\right) \mathbf{A}\left(\mathbf{t}+\frac{\tau}{2}\right) \mathbf{d t} .
$$

Equation (7), reflecting the relation between the spectrum density $|\mathbf{S}(\omega)|^{2}$ and the auto-correlation function $\mathbf{R}(\tau)$, means that the Wigner time-frequency distribution can be represented as

$$
W(t, \omega)=\frac{1}{2 \pi} \int_{-\infty}^{\infty} R_{L}(t, \tau) \exp [-i \omega \tau] d \tau
$$

where $\mathbf{R}_{\mathbf{L}}(\mathbf{t}, \tau)$ is now so-called local auto-correlation function, which depends on time. The symmetric form for this function can be written as

$$
\mathbf{R}_{\mathbf{L}}(\mathbf{t}, \tau)=\mathbf{A}^{*}\left(\mathbf{t}-\frac{\tau}{2}\right) \mathbf{A}\left(\mathbf{t}+\frac{\tau}{2}\right)
$$

see the last expression in Eq.(8); it leads to the joint Wigner distribution. To have conventional relation $\mathbf{R}(\tau)=\mathbf{R}^{*}(-\tau)$ for the auto-correlation function $\mathbf{R}(\tau)$ one has to require $\mathbf{R}_{\mathbf{L}}(\mathbf{t}, \tau)=\mathbf{R}_{\mathbf{L}}{ }^{*}(\mathbf{t},-\tau)$ for the local auto-correlation function as well.

\section{4 . THE WIGNER TIME-FREQUENCY DISTRIBUTION FOR A GAUSSIAN PULSE}

The complex amplitude of a solitary optical pulse with Gaussian shape of envelope can be written as

$$
A_{G}(t)=A_{0} \exp \left[-\frac{(1+i b) t^{2}}{2 T^{2}}\right]
$$

where $\mathbf{A}_{\mathbf{0}}$ is the real-valued amplitude, $\mathbf{T}$ is the Gaussian pulse half-width measured at a level of $\mathbf{1} / \mathbf{e}$ for the intensity contour and $\mathbf{b}$ is the parameter of frequency modulation, i.e. the frequency chirp. In this case, the joint Wigner timefrequency distribution with $\mathbf{A}_{\mathbf{0}}=\mathbf{1}$, see Eq.(5), is given by 


$$
\mathbf{W}_{G}(t, \omega)=\frac{T}{\sqrt{\pi}} \exp \left[-\frac{\mathbf{t}^{2}}{T^{2}}-\left(\omega T+\frac{b t}{T}\right)^{2}\right] .
$$

The Wigner distribution (12) for the Gaussian pulse is positive-valued. When $\mathbf{T}=\mathbf{1}$ and $\mathbf{b}=\mathbf{0}$, Eq.(12) gives the distribution, which is symmetrical relative to repositioning the variables $\mathbf{t}$ and $\boldsymbol{\omega}$. With decreasing the parameter $\mathbf{b}$, the energy distribution concentrates in a bandwidth corresponding the chirp-free spectrum whose center lies along the line $\omega=\mathbf{b t} / \mathbf{T}^{\mathbf{2}}$. Two examples of the time-frequency distribution $\mathbf{W}_{\mathbf{G}}(\mathbf{t}, \omega)=\pi^{-1 / 2} \exp \left[-\mathbf{t}^{2}-(\omega+\mathbf{b} \mathbf{t})^{2}\right]$, defined by Eq.(12) with $\mathbf{T}=\mathbf{1}$ are presented in Fig.1.

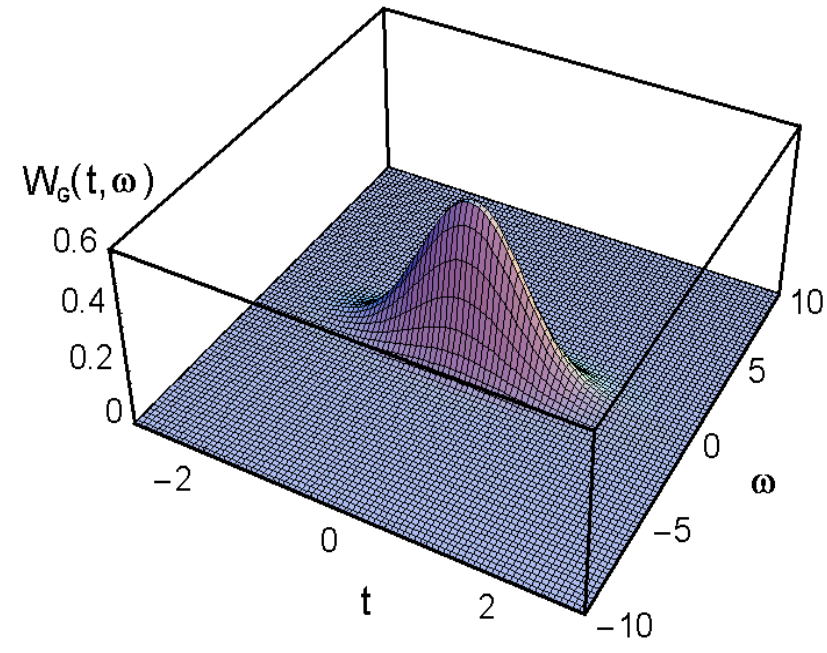

a.

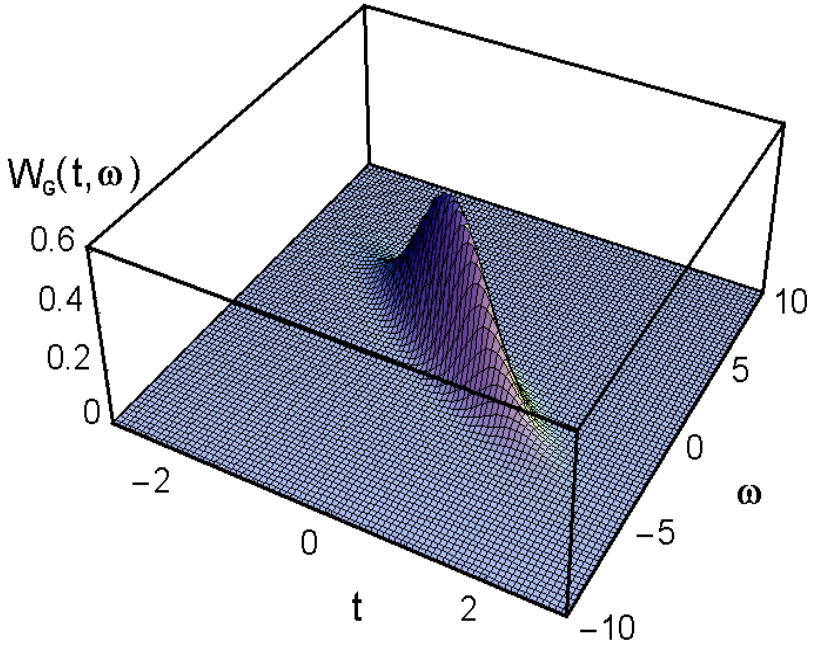

b.

Figure 1. The Wigner time-frequency distributions for the Gaussian pulses with $\mathbf{T}=\mathbf{1}$ and the varying parameter $\mathbf{b}:$ (a) $\mathbf{b}=\mathbf{0}$ and (b) $\mathbf{b}=\mathbf{2}$.

Integrations of Eq.(F2) give the partial one-dimensional Wigner distributions for a Gaussian pulse over the time or frequency separately

$$
\text { a) }\left|\mathbf{A}_{\mathbf{G}}(\mathbf{t})\right|^{2}=\int_{-\infty}^{\infty} \mathbf{W}_{\mathbf{G}}(\mathbf{t}, \omega) \mathrm{d} \omega=\exp \left(-\frac{\mathbf{t}^{2}}{\mathbf{T}^{2}}\right) \text {, b) }\left|\mathbf{S}_{\mathrm{G}}(\omega)\right|^{2}=\int_{-\infty}^{\infty} \mathbf{W}_{\mathbf{G}}(t, \omega) \mathbf{d} \mathbf{t}=\frac{\mathbf{T}^{2}}{\sqrt{1+\mathbf{b}^{2}}} \exp \left(-\frac{\mathbf{T}^{2} \omega^{2}}{1+\mathbf{b}^{2}}\right)
$$

It is seen from Eq.(13b) that to reach a level of $\mathbf{1} / \mathbf{e}$ one need vary the variable $\omega$ from $-\mathbf{T}^{-\mathbf{1}} \sqrt{\mathbf{1 + \mathbf { b } ^ { \mathbf { 2 } }}}$ to $\mathbf{T}^{-1} \sqrt{\mathbf{1 + \mathbf { b } ^ { 2 }}}$, so that the variation $\Delta \omega=\mathbf{T}^{-1} \sqrt{1+\mathbf{b}^{2}}$ means actually the half-width of the spectral contour at a level of $\mathrm{e}^{-\mathbf{1}}$. Thus, one can determine the product

$$
\Delta \omega T=\sqrt{1+b^{2}} .
$$

In the particular case of $\mathbf{b}=\mathbf{0}$ (i.e. in the absence of the frequency chirp or the phase modulation), one yields $\Delta \omega \mathbf{T}=\mathbf{1}$ for the Gaussian pulse. Nevertheless, in general case, $\mathbf{b} \gg \mathbf{1}$, so the product $\Delta \omega \mathbf{T}$ can far exceed unity. 


\section{MEASURING THE TRAIN-AVERAGE PARAMETERS OF PICOSECOND OPTICAL PULSES IN HIGH-REPETITION-RATE TRAINS}

In many cases, for example, with the investigations of evolving the optical solitons in active and passive waveguide structures, a simple method is frequently required for measuring current time-frequency parameters of low-power picoand subpicosecond optical pulses traveling in high-repetition-rate trains. Most widely used is a method based on the formation of a train-average auto-correlation function of the field strength, which is coupled through the Fourier transform with the spectral power density. From the recorded power spectral density, one can determine an average width of the radiation spectrum. However, in this case, information on the average field phase is lost and it is impossible to determine the time variation of the field amplitude $\mathbf{A ( t )}$. Exact determination of the train-average pulse duration from the width of the radiation spectrum is only possible when the shape of pulse envelope is known a priori and, in addition, the pulse spectrum is limited ${ }^{3}$. An approximate estimation of the pulse duration is also correct, if the frequency chirp is sufficiently small ${ }^{\mathbf{4}}$. Here, we demonstrate an opportunity of providing experimental conditions, under which the train-average auto-correlation function of the field strength can serve as a source of exact and reliable information on the average values of both duration and frequency chirp of a low-power optical pulses traveling in highrepetition-rate trains. As usually, let us proceed from the assumption that all pulses in a train are identical pulses with a Gaussian envelope, see Eq.(11), with the amplitude $\mathbf{A}_{\mathbf{0}}=\sqrt{\mathbf{P}}$, where $\mathbf{P}$ is the incoming pulse peak power. As it was listed above for a Gaussian envelope, the relationships between the train-average pulse parameters $\mathbf{T}$ and $\mathbf{b}$ and the width $\tau_{\mathbf{0}}$ of the corresponding auto-correlation function, measured on a level of $\mathbf{1} / \mathbf{e}$ for the intensity contour, are given by

$$
\tau=\tau_{0}=2 \mathrm{~T} / \sqrt{1+\mathbf{b}^{2}}
$$

Usually, the real-time auto-correlation function of the field strength averaged over a train of optical pulses is obtained with a scanning Michelson interferometer ${ }^{4,5}$, which allows measuring the value of $\tau_{\mathbf{0}}$. However, information on the width $\tau_{\mathbf{0}}$ of the field strength auto-correlation function is insufficient to determine the time-frequency parameters of pulse train. That is why one can propose performing two additional measurements of the auto-correlation function width with the help of a scanning Michelson interferometer. During the second and third measurements, supplementary optical components, changing the parameters $\mathbf{T}$ and $\mathbf{b}$ in a predetermined way but not influencing the envelope of the investigated pulses, should be placed in front of the beam-splitting mirror of the interferometer. The auto-correlation function widths $\tau_{\mathbf{m}} \quad(\mathbf{m}=\mathbf{1 , 2})$ obtained from the repeated measurements are coupled with the new values of the pulse duration $\mathbf{T}_{\mathbf{m}}$ and the frequency chirp $\mathbf{b}_{\mathbf{m}}$ by Eq.(15). One can assume that $\mathbf{T}_{\mathbf{m}}=\boldsymbol{\alpha}_{\mathbf{m}} \mathbf{T}_{\mathbf{0}}$ and $\mathbf{b}_{\mathbf{m}}=\mathbf{b}_{\mathbf{0}}+\boldsymbol{\beta}_{\mathbf{m}}$, where $\mathbf{T}_{\mathbf{0}}$ and $\mathbf{b}_{\mathbf{0}}$ are unknown values of the parameters $\mathbf{T}$ and $\mathbf{b}$, while the quantities $\alpha_{\mathbf{m}}$ and $\beta_{\mathbf{m}}$ are determined by supplementary optical components. Using the above-noted relations, one can write two different algebraic quadratic equations for a quantity of $\mathbf{b}_{\mathbf{0}}$. The corresponding solutions are given by a pair of the following formulas

$$
b_{0}=\left(q_{m} \alpha_{m}^{2}-1\right)^{-1}\left[\beta_{m} \pm \sqrt{q_{m} \alpha_{m}^{2}\left(\beta_{m}^{2}+2\right)-\left(q_{m}^{2} \alpha_{m}^{4}+1\right)}\right]
$$

where $\mathbf{q}_{\mathbf{m}}=\tau_{\mathbf{0}}^{\mathbf{2}} / \tau_{\mathbf{m}}^{\mathbf{2}}$ and $\tau_{\mathbf{m}}$ is the width of the field strength auto-correlation function obtained without supplementary optical components. For $(\mathbf{m}=\mathbf{1 , 2})$, Eq.(16) gives four values of $\mathbf{b}_{\mathbf{0}}$, of which two coincide with each other and correspond to just the true value of the train-average frequency chirp of the pulses. The proposed measurement method allows one to determine not only the value, but the sign of the frequency chirp as well, which is often impossible even with the help of substantially more complicated methods, such as, for example, the method described in ${ }^{6}$. Once the pulse frequency chirp $\mathbf{b}_{\mathbf{0}}$ is determined, one can use formula (16) to calculate the pulse duration $\mathbf{T}$ by using $\tau_{\mathbf{0}}$ and $\mathbf{b}=\mathbf{b}_{\mathbf{0}}$. For the supplementary electronically controlled optical component, one can propose exploiting a specific device based on an InGaAsP single-mode traveling-wave semiconductor heterolaser, which is quite similar to a saturable-absorber laser with clarified facets ${ }^{7}$. 


\section{EXPERIMENTAL STUDIES}

Semiconductor lasers have a broad gain band (about $\Delta v \approx 10^{13} \mathrm{~Hz}$ ), so that by this is meant that their operation in the regime of active mode-locking makes it possible to expect generating ultra-short optical pulses with a duration of about $\tau_{\mathbf{0}} \approx \mathbf{1} / \Delta v$ lying in a picosecond time range. Generally, the active mode-locking process provides shaping stable trains of wave packets with rather good reproducibility from pulse to pulse. Recently, this regime has been practically realized utilizing a periodic modulation of gain inherent in the active medium through injecting the pump current with a frequency equal or multiple to the frequency spacing between longitudinal modes of the laser cavity. Within this discussion, the single-mode InGaAsP/InP semiconductor heterolasers are considered. They have been designed with one antireflection-coated facet and an external single-mode optical-fiber cavity. To obtain the shortest possible optical pulses the facets of semiconductor crystals facing the fiber cavity were coated via deposition of a $\mathrm{SiO}_{2}$-film, so that the reflection coefficient was typically less than $1 \%$. An external cavity was made of a single-mode silica optical fiber with the refractive index $\mathbf{n} \approx \mathbf{1 . 5}$ and the length $\mathbf{L} \approx \mathbf{1}$ meter with an additional mirror at its far end, providing the optical feedback. The corresponding feedback factor was estimated by $15 \%$ due to about $\mathbf{4 0} \%$-efficiency of exiting the light radiation in that optical fiber by semiconductor laser structure with the refractive index $\mathbf{n}_{\mathbf{S}} \approx \mathbf{3 . 3}$. The fiber cavity length $\mathbf{L}$ corresponded to the frequency spacing about $\mathbf{f}_{\mathbf{0}} \approx 100 \mathrm{MHz}$ between its longitudinal optical modes because of $\mathbf{f}_{\mathbf{0}}=\mathbf{c} /(\mathbf{2 n})$, where $\mathbf{c}$ is the light velocity. The scheme of our experiments is presented in Fig.2.

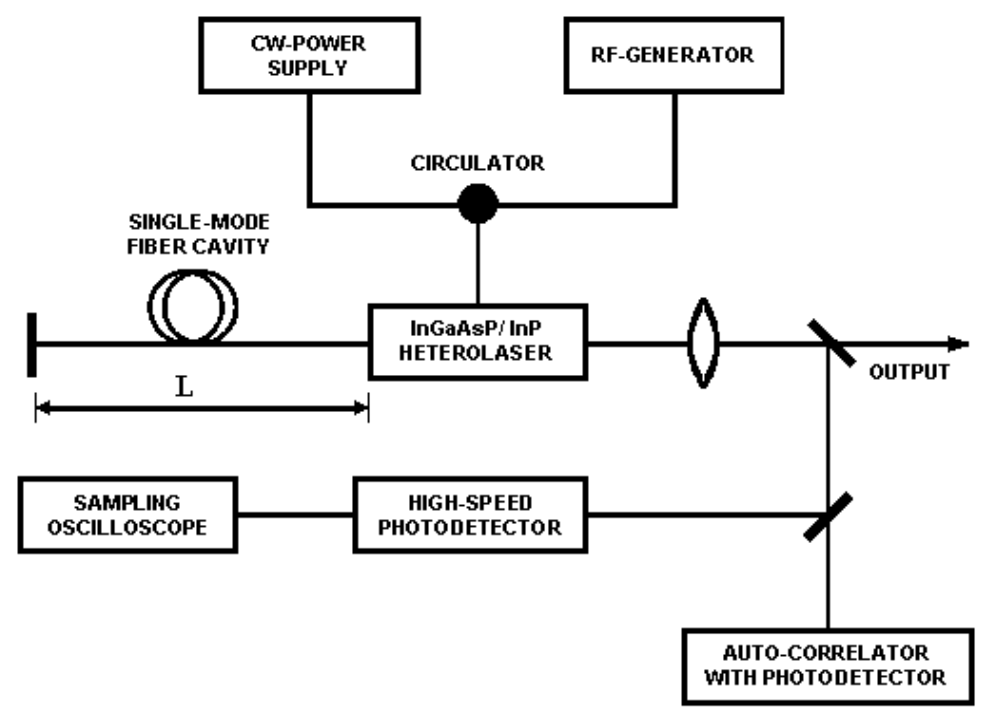

Figure 2. Schematic arrangement of the experimental set-up.

Periodic modulation of optical losses in a cavity was provided through modulating the pump current from an external source of the electronic sinusoidal RF-signal within the frequency range $400-800 \mathrm{MHz}$. The electronic port of semiconductor heterolaser was matched with a $50-\mathrm{Ohm}$ output of that source via specially designed strip-line waveguiding circuit. The regular operation of semiconductor heterolasers was provided by thermo-stabilizing system at a temperature of $16^{0} \mathrm{C}$ with an accuracy of $\pm 0.2^{0} \mathrm{C}$. The regime of operation was controlled by the diffractive optical spectrometer. Figures 3 illustrate profiles of light radiation spectra at the wavelength $\lambda=\mathbf{1 3 2 0} \mathrm{nm}$ without an external RF-modulation as well as with periodic RF-modulation applied at the semiconductor heterolaser, i.e. in the active mode-locking regime. The extended spectrum width within the active mode-locking regime was estimated by

about $\Delta \lambda \approx \mathbf{1 0 0} \mathbf{A}$. In the frequency domain, this estimation gives $\Delta v=(\Delta \lambda) \mathbf{c} / \lambda^{2} \approx \mathbf{1 . 7 2} \mathrm{THz}$ that makes it possible to expect generating trains of ultra-short optical pulses with characteristic durations lying in the picosecond range. A bit rugged profile inherent in the spectrum in Fig. $3 \mathrm{~b}$ is affected evidently by the presence of the laser diode cavity by itself and connected with residual reflections from the coated diode facet, which is facing the fiber cavity. 


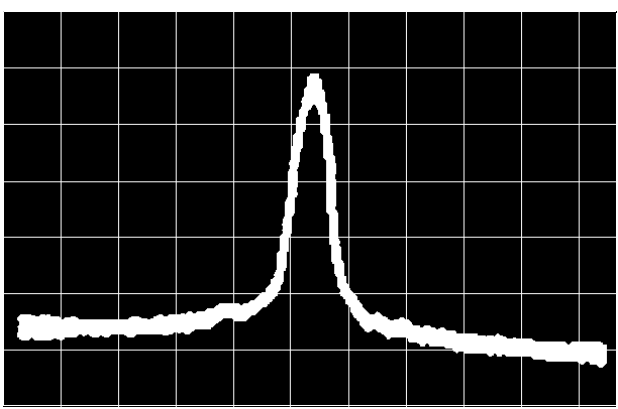

a.

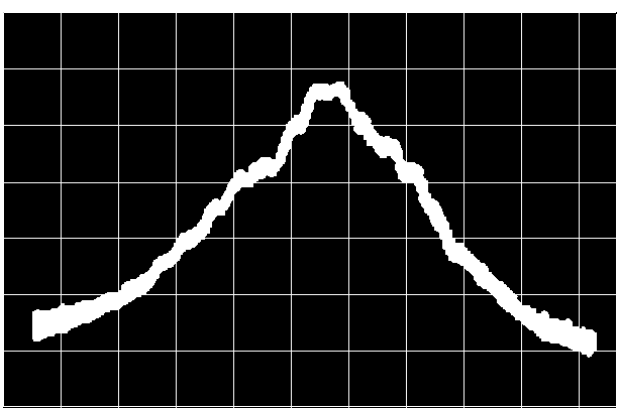

b.

Figure 3. Radiation spectra inherent in semiconductor heterolaser operating at the wavelength $\lambda=\mathbf{1 3 2 0} \mathrm{nm}$ :

(a) without an external modulation; (b) with an external sinusoidal modulation, i.e. in the active mode-locking regime.

Measuring the time-frequency parameters were carried out exploiting the modified interferometric technique described in ${ }^{7}$. During the performed proof-of-principle experiments, oriented on shaping stable trains of rather powerful (about $1 \mathrm{~W}$ in a peak) picosecond optical pulses with predictable pulse parameters and with the repetition frequency multiple to the frequency spacing of longitudinal optical modes in fiber cavity, an opportunity of estimating the train-average pulse duration as well as the train-average frequency chirp. The active mode-locking regime on multiple repetition frequencies can be associated with the cases of circulating more than one optical pulse in a long-haul cavity. A number of the circulating optical pulses $\mathbf{N}$ can be estimated as $\mathbf{N}=\mathbf{2 n f} \mathbf{L} / \mathbf{c}$, and experimentally the cases with $\mathbf{N}=\mathbf{1 - 8}$ had been successfully realized. It can be noted that the interferogram widths, measured on a level of $1 / \mathbf{e}$ for the intensity contour, were decreasing from $\mathbf{1 2 . 2}$ ps to 3.9 ps as the number $\mathbf{N}$ was growing from $\mathbf{1}$ to $\mathbf{8}$. The absolute frequency bandwidth, being available for the observation of mode-locking, was varying in the range $\mathbf{0 . 2}-\mathbf{0 . 5} \mathrm{MHz}$, so that the relative frequency locking band was a little bit less than $\mathbf{1 0}^{\mathbf{- 3}}$.

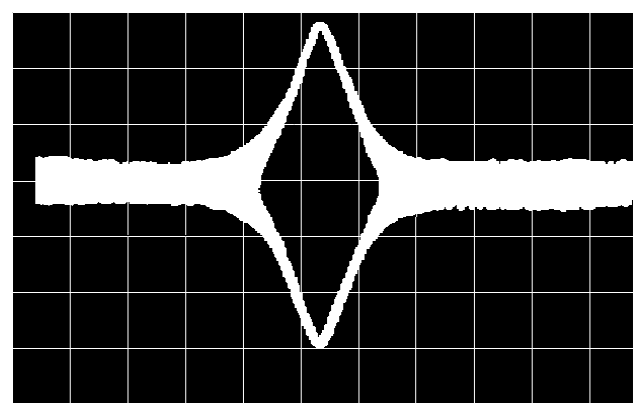

a.

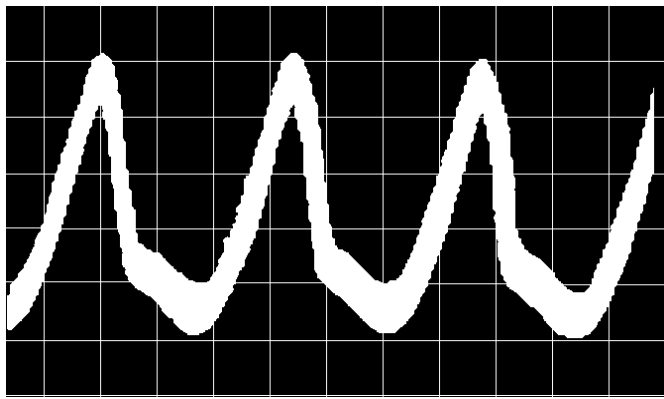

b.

Figure 4. The digitized oscilloscope traces related to a regular pulse train: (a) the train-average auto-correlation function; the pulse width of this interferogram, measured on a level of $1 / \mathbf{e}$ for the intensity contour, was estimated by 4.4 ps;

(b) the output signal from a high-speed photodetector; a train of the same ultra-short optical pulses with the repetition frequency $\mathbf{f} \approx \mathbf{7 1 8} \mathrm{MHz}$ was detected with the time resolution of about $\mathbf{3 0 0} \mathrm{ps}$.

Figure 4a represents the digitized interferogram of the second order auto-correlation function for a high-repetition-rate train of optical pulses; the width of this interferogram was estimated by 4.4 ps, while Fig.4b shows the digitized oscilloscope trace for a train of ultra-short pulses with the repetition frequency $\mathbf{f} \approx \mathbf{7} \mathbf{f}_{\mathbf{0}}=\mathbf{7 1 8} \mathrm{MHz}$, which was identified as the most stable during the experiments performed. The parameter $\mathbf{b}$, related to the frequency chirp, was estimated with applying the above-mentioned technique by $\mathbf{b} \approx \mathbf{1 . 4 6} \cdot \mathbf{1 0}^{\mathbf{- 4}}$. This is a train of picosecond pulses detected with the time resolution of about $\mathbf{3 0 0} \mathrm{ps}$, which is associated with the transfer function of a high-speed photodetector exploited. The off-duty ratio for optical pulses depicted in Fig.4b is in correspondence to the ration between the repetition period $\mathbf{1} / \mathbf{f}$ and the above-mentioned time resolution of that high-speed photodetector. 


\section{CHARACTERIZING OPTICAL PULSES}

In the active mode-locking regime, optical pulses are self-reproducing after each path through the cavity. Restoration of pulse parameters is conditioned by properties of the active medium, and because the cavity exhibits an optical dispersion, one of the necessary conditions for reproducibility of pulses is the presence of frequency chirp. Presently known mechanisms of interacting optical pulses with semiconductors allow us to simplify the theoretical model of shaping an ultra-short pulse with the complex field amplitude $\mathbf{E}(\mathbf{t})=\mathbf{A}(\mathbf{t}) \exp \left(\mathbf{i} \omega_{0} \mathbf{t}\right)+\mathbf{c} \cdot \mathbf{c}$. in a heterostructure.

The pulse, grown during the process of active mode-locking, has a Gaussian shape and can be described by Eq.(11). The pulse width, measured on a level of $\mathbf{1} / \mathbf{e}$ for the intensity contour, is given by ${ }^{8}$

$$
T=(g \mathbf{m})^{-1 / 4}\left(\omega_{m} \omega_{S}\right)^{-1 / 2}
$$

where $\mathbf{g}$ is the maximal gain at $\mathbf{t}=\mathbf{0}, \mathbf{m}$ is the factor of external modulation of the losses in a cavity, $\omega_{\mathbf{m}}$ is the external modulation frequency, and $\omega_{\mathrm{S}}$ is the gain contour width. Finally, the frequency chirp can be expressed as ${ }^{8}$

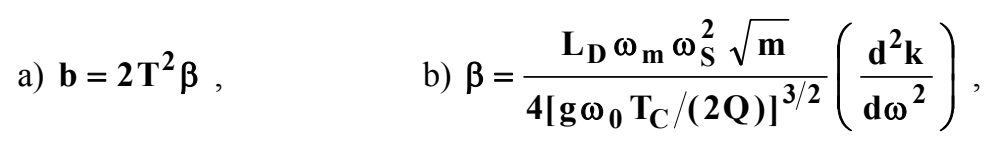

where $\beta$ is the dimensional factor of frequency chirp, $\mathbf{L}_{\mathbf{D}}$ is the length of high-dispersion components (for example, the laser crystal), $\boldsymbol{\omega}_{\mathbf{0}}$ is the central frequency of emission, $\mathbf{Q}$ is the quality factor inherent in a cavity, $\mathbf{T}_{\mathbf{C}}$ is the transit time of a pulse through a cavity, and $\mathbf{k}$ is the wave number. In fact, Eqs.(17) and (18) can be practically used to estimate the parameters of the optical pulses generated.

Using the values characteristic of the experiments: $\mathbf{g}=\mathbf{3}, \mathbf{m}=\mathbf{0 . 2 5}, \omega_{\mathrm{m}}=\mathbf{2} \pi \cdot \mathbf{7 1 8} \cdot \mathbf{1 0} \mathbf{\mathrm { rad }} / \mathrm{s}$, and $\omega_{\mathrm{S}}=\mathbf{2} \pi \cdot \mathbf{1 0} \mathbf{1 3}^{\mathbf{1 3}}$ $\mathrm{rad} / \mathrm{s}$, one can obtain $\mathbf{T} \approx \mathbf{2 . 7 3}$ ps from Eq.(17), which can be considered as rather good agreement with the experimental data. The frequency chirp that arises within establishing the self-reproducing pulses can be estimated with Eq.(18). For $\mathbf{L}_{\mathbf{D}} \approx \mathbf{0 . 5} \mathrm{mm}, \boldsymbol{\omega}_{\mathbf{0}}=\mathbf{2 . 1} \cdot \mathbf{1 0} \mathbf{1 5}^{\mathrm{rad} / \mathrm{s}}\left(\right.$ at $\lambda=\mathbf{1 3 2 0} \mathrm{nm}$ ), $\mathbf{T}_{\mathbf{C}}=\mathbf{1 0} \mathbf{8}^{-\mathbf{8}} \mathrm{s}, \mathbf{Q}=\mathbf{1 0} \mathbf{5}^{\mathbf{5}}$, and $\left(\mathbf{d}^{2} \mathrm{k} / \mathbf{d} \omega^{2}\right)=\mathbf{3 . 7} \cdot 10^{-24} \mathrm{~s}^{2} / \mathrm{m}$, one can obtain $\beta=\mathbf{7 . 3} \cdot 10^{18} \mathrm{~s}^{-2}$ from Eq.(18b). Nevertheless, this dimensional magnitude of the estimated frequency chirp is relatively small, because one can find from Eq.(18a) in dimensionless values that $\mathbf{b} \approx \mathbf{0 . 8 4} \cdot \mathbf{1 0}^{-\mathbf{4}} \ll \mathbf{1}$. In practically reasonable assumption that the envelopes of optical pulses under consideration can be described rather adequately by Gaussian functions, these estimations make it possible to create the corresponding theoretical version of Wigner time-frequency distribution with the above-calculated parameters $\mathbf{T}$ and $\mathbf{b}$. Together with this, the experimental version of similar time-frequency distribution can be designed with experimentally obtained parameters $T \approx \mathbf{2 . 2}$ ps and $\mathbf{b} \approx \mathbf{1 . 4 6} \cdot \mathbf{1 0}^{-\mathbf{4}}$ in the same approximation by Gaussian functions. The resulting plots of two Wigner distributions for the Gaussian-like optical pulses, obtained from estimations and from experiment, are shown in Fig.5.

\section{CONCLUSION}

A novel approach to the characterization of low-power bright picosecond optical pulses with an internal frequency modulation in both time and frequency domains in practically important cases of exploiting semiconductor heterolasers operating in near-infrared range in the active mode-locking regime has been presented. This approach is oriented to using the joint Wigner time-frequency distributions. Similar distributions can be created for this regime within exploiting the progressed interferometric briefly described above. The modified scanning Michelson interferometer has been chosen for obtaining the field-strength auto-correlation functions. In fact, we have presented the key features of a new experimental technique for accurate and reliable measurements of the train-average temporal width and the frequency chirp of picosecond optical pulses in high-repetition rate trains. This technique makes it possible to find the 
parameters needed for reconstructing the joint Wigner distributions inherent in optical pulses. The InGaAsP/InPheterolasers, operating at $\mathbf{1 3 2 0} \mathrm{nm}$ wavelength range, have been used within the experiments. When the optical signal consists of contiguous pulses with the repetition frequency close to $1 \mathrm{GHz}$, conditioned by operating semiconductor lasers in the active mode-locking regime, typical requirements for measurements and operating with the Wigner distributions have been satisfied, so that the train-average pulse parameters have been successfully characterized.

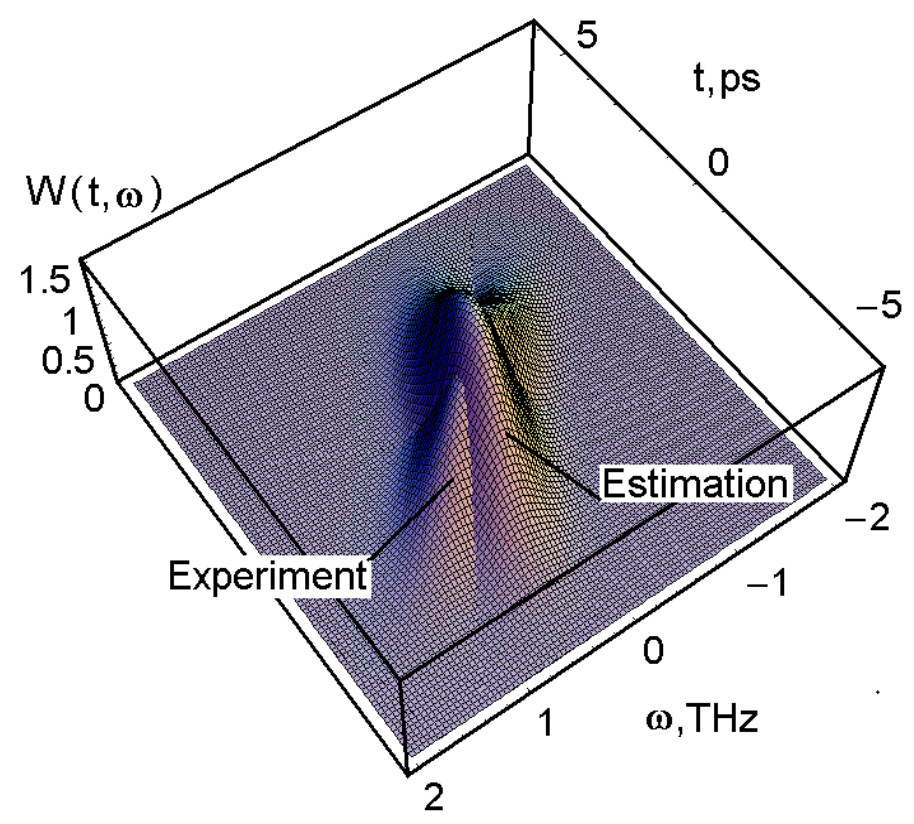

Figure 5. A pair of the Wigner time-frequency distribution for the Gaussian pulses obtained from the performed estimation with $\mathbf{T}=\mathbf{2 . 7 3}$ ps and the $\mathbf{b}=\mathbf{0 . 8 4} \cdot \mathbf{1 0}^{-\mathbf{4}}$ as well as from the experiment with $\mathbf{T}=\mathbf{2 . 2}$ ps and the $\mathbf{b}=\mathbf{1 . 4 6} \cdot \mathbf{1 0}-\mathbf{4}$.

\section{ACKNOWLEGEMENT}

This work has been financially supported by the CONACyT, Mexico (project \#61237-F).

\section{REFERENCES}

1. G. P .Agrawal and N.K.Dutta, Semiconductor lasers. 2-nd Ed. (Van Nostrand Reinhold, New-York, 1993).

2. L. Cohen. Time-frequency distributions - a review. Proc. IEEE., vol.77, 941-981 (1989).

3. E. P. Ippen and C.V. Schenk. "Picosecond Techniques and Applications" in Ultrashort Light Pulses, Ed. S.Shapiro (Springer, Heidelberg, 1977).

4. A. S. Shcherbakov. Synchronization of a radio-interferometer by the high-repetition-rate picosecond solitons. Tech. Phys. Lett., vol.19, 615-616 (1993).

5. J. Herrmann and B. Wilhelmi. Laser fur Ultrakurze Lichtimpulse. (Akademi-Verlag, Berlin, 1984).

6. K. Nagamuna, K. Mogi, and H. Yamada. General method for ultrashort light pulse chirp measurement. IEEE J. Quantum Electron., vol.25, 1225-1233 (1989).

7. A. S. Shcherbakov, A. L. Munoz Zurita, A.Yu.Kosarsky, and J Campos Acosta. Determining the time - frequency parameters of low-power bright picosecond optical pulses using interferometric technique. OPTIK - Int. J.Electron. Opt.(Elsevier, Germany); doi:10.1016/j.ijleo.2008.07.033.

8. J. P. van der Ziel. The mode-locking in semiconductor lasers. // Semiconductors \& Semimetals, Vol. 22 ( Lightwave Communication Technology), Volume Editor W.T. Tsang (Academic Press, Orlando, 1985) Chapter 1. 